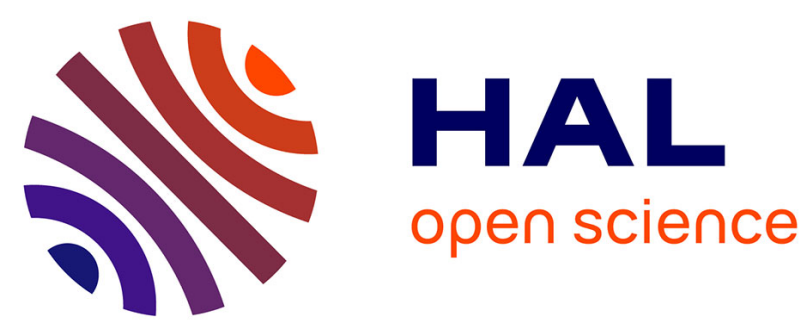

\title{
Lectin recognition and hepatocyte endocytosis of GalNAc-decorated nanostructured lipid carriers
}

Laura Gauthier, Mireille Chevallet, Francois Bulteau, Michel Thépaut, Pascale Delangle, Franck Fieschi, Corinne Vivès, Isabelle Texier, Aurélien Deniaud, Christelle Gateau

\section{To cite this version:}

Laura Gauthier, Mireille Chevallet, Francois Bulteau, Michel Thépaut, Pascale Delangle, et al.. Lectin recognition and hepatocyte endocytosis of GalNAc-decorated nanostructured lipid carriers. Journal of Drug Targeting, 2021, 29 (1), pp.99-107. 10.1080/1061186X.2020.1806286 . hal-02969595

\section{HAL Id: hal-02969595 https://hal.science/hal-02969595}

Submitted on 5 Nov 2021

HAL is a multi-disciplinary open access archive for the deposit and dissemination of scientific research documents, whether they are published or not. The documents may come from teaching and research institutions in France or abroad, or from public or private research centers.
L'archive ouverte pluridisciplinaire HAL, est destinée au dépôt et à la diffusion de documents scientifiques de niveau recherche, publiés ou non, émanant des établissements d'enseignement et de recherche français ou étrangers, des laboratoires publics ou privés. 


\section{Lectin recognition and hepatocyte endocytosis of GalNAc-decorated Nanostructured Lipid Carriers}

Laura Gauthier, ${ }^{\mathrm{a}, \mathrm{b}}$, Mireille Chevallet ${ }^{\mathrm{c}}$, Francois Bulteau ${ }^{\mathrm{d}}$, Michel Thépaut ${ }^{\mathrm{d}}$, Pascale Delangle $^{\mathrm{a}}$, Franck Fieschi ${ }^{\mathrm{d}}$, Corinne Vivès ${ }^{\mathrm{d}}$, Isabelle Texier ${ }^{\mathrm{b} *}$, Aurélien Deniaud ${ }^{\mathrm{c} *}$ and Christelle Gateau ${ }^{\mathrm{a} *}$

${ }^{a}$ Univ. Grenoble Alpes, CEA, CNRS, IRIG-SyMMES, F-38000 Grenoble, France; ${ }^{b}$ Univ. Grenoble Alpes, CEA, LETI-DTBS, F-38000 Grenoble, France; ' Univ. Grenoble Alpes, CNRS, CEA, IRIG - Laboratoire de Chimie et Biologie des Métaux, F-38000 Grenoble, France; ${ }^{d}$ Univ. Grenoble Alpes, CEA, CNRS, IRIG - Institut de Biologie Structurale, F38000

* Correspondence: christelle.gateau @ cea.fr; Tel.: +33438789822

* Correspondence: aurelien.deniaud@cea.fr; Tel.: +334 38789651

* Correspondence: isabelle.texier-nogues@cea.fr; Tel.: +33438784670 


\begin{abstract}
Liver is the main organ for metabolism but is also subject to various pathologies, from viral, genetic, cancer or metabolic origin. There is thus a crucial need to develop efficient liver-targeted drug delivery strategies. Asialoglycoprotein receptor (ASGPR) is a C-type lectin expressed in the hepatocyte plasma membrane that efficiently endocytoses glycoproteins exposing galactose $(\mathrm{Gal})$ or Nacetylgalactosamine (GalNAc). Its targeting has been successfully used to drive the uptake of small molecules decorated with three or four GalNAc, thanks to an optimization of their spatial arrangement. Herein, we assessed the biological properties of highly stable nanostructured lipid carriers (NLC) made of FDAapproved ingredients and formulated with increasing amounts of GalNAc. Cellular studies showed that high density of GalNAc was required to favour hepatocyte internalization via the ASGPR pathway. Interaction studies using surface plasmon resonance and the Macrophage Galactose-Lectin as GalNAc-recognizing lectin confirmed the need of high GalNAc density for specific recognition of these NLC. This work is a first step for the development of efficient nanocarriers for prolonged liver delivery of active compounds.
\end{abstract}

Keywords: N-acetylgalactosamine; Surface functionalization; Nanostructured lipid carriers; Asialoglycoprotein receptor; Macrophage galactose-lectin; Hepatocytes; Targeted drug delivery. 


\section{Introduction}

Liver insures several vital functions, such as nutrients or xenobiotics metabolism. Liver damages potentially lead to cirrhosis, hepatic failure, or liver cancer. There are more than a hundred liver diseases associated with alcohol consumption (alcohol-related liver disease), viral infection (hepatitis) or genetic diseases (Wilson's disease). Given the number of liver diseases and their increasing incidence, it is crucial to develop hepatic drug-delivery systems to maximize therapeutic efficiency of established active drugs.

A gold road to specifically target a tissue is to use a receptor specifically expressed on the corresponding cell type as a gateway. Asialoglycoprotein receptor (ASGPR) is mainly found in the plasma membrane of hepatic cells $[1,2]$. It is a C-type lectin that possesses a specific carbohydrate recognition domain (CRD) responsible for the recruitment of galactose (Gal) and $\mathrm{N}$-acetylgalactosamine (GalNAc) in a calciumdependent manner. It has thus been extensively studied as a promising candidate for drug delivery into hepatocytes [1-3]. It is responsible for the recognition, binding and clearance of desialylated glycoproteins, i.e. Gal and GalNAc terminated [4]. ASGPR is an hetero-oligomer made of two highly homologous polypeptides $(\mathrm{H} 1$ and $\mathrm{H} 2,58 \%$ sequence homology). Heterotrimer and heterotetramer have been described in different species with 2:1 and 3:1 stoichiometric ratios, respectively [5]. ASGPR H1 and H2 consist in a $\mathrm{N}$-terminal motif involved in endocytosis mediated by clathrin-coated domains, a transmembrane segment, and an extracellular C-terminal CRD [2,6]. ASGPR recognizes specifically Gal and GalNAc with an affinity for GalNAc approximately 50fold higher than for Gal [7-9]. Besides, its oligomerization clusters CRDs together, leading to a very high affinity for trivalent or tetravalent glycans, $\sim 100,000$ times higher than their monomeric CRD [5]. Hence, exploiting multivalent interactions has proven to be a promising targeting strategy since it improves both the efficiency and the selectivity 
of the recognition process [4,10-15]. A number of synthetic ligands have been designed and have demonstrated that the geometry of the multivalent architecture is a keyparameter, determining the binding affinity for ASGPR [16-19]. Among these ligands, the compound described by Khorev et al [16], bearing three GalNAc arranged at the vertices of a triangle with sides averaging $20 \AA$, exhibited a specific and high affinity binding to ASGPR. In the context of Wilson's disease, we have previously reported two series of bifunctional molecules that combine a copper chelating unit and ASGPR targeting motifs [20-23]. They displayed three or four GalNAc units anchored on a tripod or a cyclodecapeptide scaffold, respectively, with flexible spacers ensuring the appropriate $20 \AA$ distances between the sugar units. Both architectures demonstrated an efficient cellular uptake in HepG2 hepatoma-derived cells by three to four orders of magnitude in comparison with monovalent GalNAc. These targeting moieties promoted efficient ASGPR-mediated uptake of $\mathrm{Cu}$ chelators and hence represented an attractive and innovative therapeutic option to treat Wilson's disease. The results corroborated the crucial role of the spatial arrangement of Gal and GalNAc units in the recognition and uptake by ASGPR. This clustering effect could also be achieved by the surface “decoration" of nanocarriers using Gal or GalNAc ligands.

Several studies have reported that the functionalization of nanoparticles by Gal or GalNAc motifs promote their efficient recognition with lectins, their internalization in hepatic cells and therefore establish their interest for liver delivery of therapeutic substances [24-32]. Among the large range of available nanocarriers, lipid-based nanoparticles, including liposomes, lipid nano-emulsions, solid lipid nanoparticles, lipid nanocapsules and nanostructured lipid nanocarriers (NLC) are of particular interest for their safety profile, drug payload ability, and up-scalable fabrication process [33-37]. For example, Hashida's group has developed $85 \mathrm{~nm}$ diameter liposomes functionalized by 0 
to $7.5 \%$ mol. of galactose $[27,28,38]$. Formulations containing $3.5 \%, 5 \%$ and $7.5 \%$ of Gal efficiently accumulated in mouse liver (approximately $85 \%$ of the injected dose against $12 \%$ for non-functionalized nanoparticles). Morille et al. designed lipid nanocapsules (110 to $180 \mathrm{~nm}$ diameter) for gene therapy, functionalized by galactose using two different types of surfactants: DSPE-PEG $_{40}-\mathrm{Gal}$ (DSPE: distearoylphosphatidylethanolamine, $\mathrm{PEG}_{40}$ : poly(ethylene) glycol with about 40 ethylene oxide units, 1 Gal unit/surfactant) and F108-(Gal) (F108: Pluronic F108, 2 Gal units/surfactant) [25]. Lectin aggregation tests (Soybean agglutinin, SBA) evidenced a specific interaction of F108-(Gal $)_{2}$ functionalized LNC three times higher than with nanocapsules functionalized by DSPE-PEG $40-$ Gal, and a significant increase (18 times) in transfection in primary rat hepatocytes, suggesting a difference in the accessibility of sugars at the nanoparticle surface. Sato et al. also published an interesting study in which they compared the pharmacokinetics and in vivo uptake in hepatocytes of bare liposomes, interacting through the ApoE-mediated pathway, versus GalNAc-decorated particles, whose hepatocyte entry was mediated by ASGPR [30]. They evidenced that the two types of nanoparticles (i.e. internalized via different pathways) presented different blood clearance and hepatocyte entry kinetics.

In a previous study, we decorated $50 \mathrm{~nm}$ diameter NLC with GalNAc monosaccharide units [39]. These nanoparticles presented several advantages [40,41], such as the biocompatibility of their FDA-approved ingredients, a small size, an easy fabrication process at large scale [42], a core composed of a mixture of liquid and solid lipids to enhance particle colloidal stability [43], and a quantified, controlled, and tunable number of GalNAc ligands [39], whereas literature studies generally assume full incorporation of targeting ligands on the nanoparticle surface. Herein, the influence of the GalNAc ligand density on the targeting capabilities of the NLC was investigated, 
combining molecular and cellular studies. We showed that NLC were efficiently internalized by hepatocytes using, at least partly, the ASGPR endocytic pathway. However, a high density of GalNAc was required for the recruitment of NLC to specific lectins.

\section{Materials and Methods}

\section{NLC formulations}

Suppocire $^{\mathrm{TM}}$ NB was purchased from Gattefossé (Saint-Priest, France), Lipoid ${ }^{\mathrm{TM}}$ S75 (soybean lecithin at $>75 \%$ phosphatidylcholine) from Lipoid (Ludwigshafen, Germany), Myrj $^{\text {TM }}$ S40 and Myrj ${ }^{\text {TM }}$ S100 (mixture of stearate and oleate-poly(ethylene glycol) surfactants with respectively 40 and 100 ethylene glycol units) and super-refined soybean oil from Croda Uniqema (Chocques, France), and DiD (1,1'-dioctadecyl-3,3,3',3'tetramethylindodicarbocyanine perchlorate) from Fisher Scientific (Les Ulis, France). The synthesis of SA-PEG $100-$ GalNAc (SA: stearic acid, PEG100: poly(ethylene glycol) with 100 ethylene glycol units) has been previously described [39]. Other chemicals were purchased from Sigma-Aldrich (Saint-Quentin-Fallavier, France).

GalNAc-NLC (formulations A-C) and Myrj ${ }^{\mathrm{TM}}$ S100-NLC (formulations A'-C') were formulated by mixing prepared lipid and aqueous phases at $50^{\circ} \mathrm{C}$ prior to ultrasonication (VCX750 Ultrasonic processor, Sonics, Newtown, USA, 5 minutes at 25\% power, $10 \mathrm{~s}$ ON, 30 s OFF), according to previously reported procedures $[39,43,44]$. The lipid phase comprised soybean oil, Suppocire ${ }^{\mathrm{TM}} \mathrm{NB}$, lecithin, and eventually DiD (800 nmol), and the aqueous phase was composed of Myrj ${ }^{\mathrm{TM}} \mathrm{S} 40$, Myrj $^{\mathrm{TM}} \mathrm{S} 100$ or SA-PEG ${ }_{100}-\mathrm{GalNAc}$ and 1X PBS buffer (10 mM phosphate, $\mathrm{pH} 7.4$, qsp $2 \mathrm{~mL})$, with ingredient quantities summarized in Table 1. The nanoparticle dispersions were then purified by dialysis 
against 1X PBS using 12-14 kDa molecular weight cutoff membranes (ZelluTrans, Roth, France).

Dynamic light scattering (DLS) was used to determine the particle hydrodynamic diameter and zeta potential (Zeta Sizer Nano ZS, Malvern Instrument, Orsay, France). Particle dispersions were diluted to $2 \mathrm{mg} \cdot \mathrm{mL}^{-1}$ of lipids in $0.22 \mu \mathrm{m}$ filtered $0.1 \mathrm{X}$ PBS and transferred in Zeta Sizer Nano cells (Malvern Instrument) before each measurement, performed in triplicate. TEM images of NLC were obtained after negative staining (2\% uranyl acetate) similarly to a previously published protocol [45]. Particle concentration (expressed in $\mathrm{mg}$ of lipids $\cdot \mathrm{mL}^{-1}$ ) was determined by weighting lyophilized dispersion samples of known volumes (taking into account the weight of buffer salts). Absorbance measurements (Cary UV-visible spectrophotometer, Les Ulis, France) at $650 \mathrm{~nm}$ performed on particle dispersions diluted to $10 \mathrm{mg} \cdot \mathrm{mL}^{-1}$ of lipids allowed the quantification of the number of DiD dyes encapsulated per NLC (encapsulation yield of DiD > 95\% for all formulations) [44].

The number of GalNAc moities grafted on the particle surface was quantified by ultrahigh-performance liquid chromatography (UPLC) separation and evaporative lightscattering detection (ELSD) as previously described [39]. For UPLC-ELSD analysis, nanoparticles were disassembled by precipitation in acetonitrile. Samples were centrifuged to pellet the lipids after precipitation. The supernatant was dissolved in acetonitrile and the samples were centrifuged once more to remove any remaining lipid. The supernatants were concentrated under reduced pressure and dissolved in a mixture of $\mathrm{CHCl}_{3} / \mathrm{MeOH} 2 / 1(\mathrm{v} / \mathrm{v})$ to yield solutions with theoretical total $1 \mathrm{mg} \cdot \mathrm{mL}^{-1}$ concentration of PEGylated surfactants (Myrj ${ }^{\mathrm{TM}}$ S40, Myrj ${ }^{\mathrm{TM}} \mathrm{S} 100$ and SA-PEG 100 -GalNAc) before analysis. Analysis was performed using an Acquity UPLC® HClass system (Waters) coupled with an Alltech 3300 Evaporating Light Scattering detector (ELSD, Grace). 
Separation of the different components was achieved using a CORTECS RP-18 column $(1.6 \mu \mathrm{m}, 150 \times 2.1 \mathrm{~mm})$. The drift tube was set at $45^{\circ} \mathrm{C}$ with a flow of $\mathrm{N}_{2}$ set at $2.0 \mathrm{~L} \cdot \mathrm{min}^{-}$ ${ }^{1}$ and a gain at 4 . The injected volume was $5 \mu \mathrm{L}$, the column temperature was set at $40^{\circ} \mathrm{C}$, and eluent flow at $0.3 \mathrm{~mL} \cdot \mathrm{min}^{-1}$. Eluents were deionized water $(\mathrm{A})$, methanol (B), and a mixture of isopropanol/acetonitrile 75/25 (v/v) (C). Gradients were: from 0 to 3 minutes: from $30 / 70 / 0 \mathrm{~A} / \mathrm{B} / \mathrm{C}$ to $10 / 90 / 0 \mathrm{~A} / \mathrm{B} / \mathrm{C}$; from 3 to 15 minutes: from $10 / 90 / 0 \mathrm{~A} / \mathrm{B} / \mathrm{C}$ to 0/100/0 A/B/C; from 15 to 22 minutes: from 0/100/0 A/B/C to 0/35/65 A/B/C; from 22 to 25 minutes: isocratic $0 / 35 / 65 \mathrm{~A} / \mathrm{B} / \mathrm{C}$; from 25 to 25.1 minute: from $0 / 35 / 65 \mathrm{~A} / \mathrm{B} / \mathrm{C}$ to 30/70/0 A/B/C; from 25.1 to 30 minutes: isocratic 30/70/0 A/B/C. Each of the standards

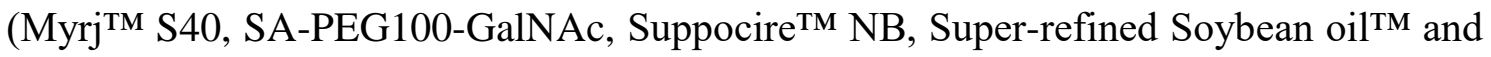
Lipoid s75 ${ }^{\mathrm{TM}}$ ) was weighed on a calibrated analytical balance and dissolved in a mixture of $\mathrm{CHCl}_{3} / \mathrm{MeOH} 2 / 1(\mathrm{v} / \mathrm{v})$ to give $1 \mathrm{mg} \cdot \mathrm{mL}^{-1}$ stock solutions. UPLC-ELSD calibrations curves were established for each ingredient based on serial dilutions of these stock solutions to conduct the identification and quantification of the different ingredients in the formulations [39].

\begin{tabular}{|c|c|c|c|c|c|c|c|}
\hline & & $\mathbf{A}$ & $A^{\prime}$ & $\mathbf{B}$ & $B^{\prime}$ & C & $C^{\prime}$ \\
\hline \multirow[t]{3}{*}{ Lipid phase } & Soybean oil & 85 & 85 & 85 & 85 & 85 & 85 \\
\hline & Wax (Suppocire NB ${ }^{\mathrm{TM}}$ ) & 245 & 245 & 245 & 245 & 245 & 245 \\
\hline & Lecithin (Lipoid ${ }^{\mathrm{TM}}$ s75) & 65 & 65 & 65 & 65 & 65 & 65 \\
\hline \multirow[t]{3}{*}{ Aqueous phase } & Myrj S40 & 327.0 & 298.3 & 241.1 & 327.0 & 298.3 & 241.1 \\
\hline & SA-PEG100-GalNAc & 18.7 & 46.7 & 105.4 & - & - & - \\
\hline & Myrj S100 ${ }^{\mathrm{TM}}$ & - & - & - & 18.7 & 46.7 & 105.4 \\
\hline
\end{tabular}

Table 1. Composition of formulations A, B, C, and A', B', C'. 


\section{Surface Plasmon Resonance}

The extracellular domains (ECD) of macrophage galactose-lectin (MGL S ECD) and DCSIGN (DC-SIGN S ECD) were produced as previously described [46,47]. Surface Plasmon Resonance (SPR) experiments were performed on a Biacore T200 on Series S Sensor Chip C1 and CM3 (GE-LifeSciences). CM3 sensor chip surface is covered by short dextrans, while $\mathrm{C} 1$ sensor chip surface is directly carboxymethylated without dextran. $\mathrm{C} 1$ presents the advantage to avoid non-specific interactions between PEG and dextran. Experiments were performed at $5 \mu \mathrm{L} \cdot \mathrm{min}^{-1}$. Strep-Tactin (IBA company) and MGL S ECD were immobilized on the flow cells using amine-coupling method. Flow cell $(\mathrm{Fc}) 1$ was prepared as reference surface. Fc1 and 2 were activated with $0.2 \mathrm{M}$ EDC / 0.05 M NHS mixture. After this step, Fc1and Fc2 were functionalized with $195 \mu \mathrm{L}$ of $170 \mu \mathrm{g} \cdot \mathrm{mL}^{-1}$ StrepTactin in $10 \mathrm{mM} \mathrm{NaOAc} \mathrm{pH} 4$, and then remaining activated groups of all Fc were blocked with $1 \mathrm{M}$ ethanolamine. After blocking, the two Fc were treated at $100 \mu \mathrm{L} \cdot \mathrm{min}^{-1}$ with $10 \mathrm{mM} \mathrm{HCl}$ to remove non-specifically bound proteins and $50 \mathrm{mM}$ $\mathrm{NaOH} / 1 \mathrm{M} \mathrm{NaCl}$. Finally, about 3100 and 200 RU of StrepTactin were immobilized on both $\mathrm{Fc}$ for $\mathrm{CM} 3$ and $\mathrm{C} 1$ surface, respectively. This procedure was repeated for the functionalization of Strep Tag II tagged MGL ECD (using $100 \mu \mathrm{g} \cdot \mathrm{mL}^{-1}$ solution) on Fc2 and enabled the functionalization of 2300 and 640 RU for CM3 and C1 surface, respectively. For CM3 experiment, an additional Fc was prepared similarly with DCSIGN ECD and enabled to reach a functionalization of $2150 \mathrm{RU}$.

To confirm the effective functionalization of the channel Fc2, a titration using BSA-GalNAc was performed. Increasing concentrations of the different formulations of NLC with different molar percentages of SA-PEG $100-$ GalNAc (Formulations A-C) or Myrj $^{\mathrm{TM}} \mathrm{S} 100$ (Formulations A'-C') were prepared in PBS, $1 \mathrm{mM} \mathrm{CaCl}_{2}, 0.05 \%$ P20 surfactant. A concentration range from $7.8 \mu \mathrm{M}$ to $2 \mathrm{mM}$ in GalNAc $\left(97 \mu \mathrm{g} \cdot \mathrm{mL}^{-1}\right.$ to 
$100 \mathrm{mg} \cdot \mathrm{mL}^{-1}$ of lipids in NLC) was used for formulations A, B and C (NLC with respectively 4.6, 13.9 and $23.3 \%$ molar percentages of $\mathrm{SA}-\mathrm{PEG}_{100}-\mathrm{GalNAc}$ ). For control NLC without GalNAc, (Formulations A', B' and C'), a lipid concentration range from $97 \mu \mathrm{g} \cdot \mathrm{mL}^{-1}$ to $100 \mathrm{mg} \cdot \mathrm{mL}^{-1}$ was also used corresponding to the lipid concentration range of the GalNAC formulations A, B and C, respectively. Experiments were performed at $20^{\circ} \mathrm{C}$ at a flow rate of $20 \mu \mathrm{L} \cdot \mathrm{min}^{-1}$. After $250 \mathrm{sec}$ association, ligand dissociation was enabled for another $250 \mathrm{sec}$. Regeneration of the surface was achieved by the injection of 25 mM EDTA, 50 mM Glycine, 0.15\% Triton pH 12.

\section{Cell culture}

HepG2 and its derivative HepG2/C3a cell lines from ATCC were grown in Minimum Essential Medium (MEM) containing L-glutamine supplemented with $10 \% \mathrm{v} / \mathrm{v}$ foetal bovine serum (FBS), $100 \mathrm{U} \cdot \mathrm{mL}^{-1}$ penicillin and $100 \mu \mathrm{g} \cdot \mathrm{mL}^{-1}$ streptomycin. Cells were cultured at $37^{\circ} \mathrm{C}$ in a humidified atmosphere with $5 \% \mathrm{CO}_{2}$.

\section{NLC uptake analysed by flow cytometry}

Cells were seeded at $1.5 \cdot 10^{5}$ cells per well in 12 -well plates 24 hours before starting NLC exposure. Five hundred $\mu \mathrm{L}$ of fresh medium containing the appropriate concentration of DiD-loaded NLC were added in the different wells for $30 \mathrm{~min}$. Then, HepG2 cells were washed three times with PBS and recovered using Trypsin-EDTA. After rinsing with complete cell culture medium, cells were centrifuged (3 min, $5000 \mathrm{rpm})$. Cell pellet was resuspended with $200 \mu \mathrm{L}$ PBS for flow cytometry analysis. Cell suspensions were kept on ice and analyzed using a Beckman Coulter FACSCalibur flow cytometer with the $635 \mathrm{~nm}$ laser excitation wavelength. Fluorescence was collected with a FL4-H detector $(661 \mathrm{~nm})$. Gating during flow analysis was based on normalized fluorescence of untreated cells. An average of 20,000 healthy cells (gated events) were analysed for each 
experimental condition, and the mean fluorescence intensity (MFI) of this population was taken as the background. The uptake of a compound into cells was evaluated by comparing the shift in MFI between untreated cells (background fluorescence) and treated cells to evaluate cellular internalization of the nanoparticles. Data analysis was performed thanks to the Cell Quest Pro software. Further analysis, such as $\mathrm{EC}_{50}$ (corresponding to the half-maximal effective concentration) calculations were done with Excel. Each experiment was performed at least three times independently.

\section{Confocal microscopy}

HepG2/C3a cells, grown overnight on glass coverslips, were incubated for 1 hour with DiD-loaded GalNAc- and Myrj ${ }^{\mathrm{TM}} \mathrm{S} 100-\mathrm{NLC}$ at a concentration of $500 \mu \mathrm{g} \cdot \mathrm{mL}^{-1}$ of lipids. Cells were then fixed with formalin (Sigma) at room temperature for $30 \mathrm{~min}$ and rinsed 3 times with PBS, cells were incubated 5 minutes with $1 \mu \mathrm{M}$ Hoechst 33342 in the last rinsing solution. Cells were finally imaged using Zeiss LSM880 inverted laser scanning confocal microscope with a 63x oil immersion objective (HCX PL APO 63.0 X 1.40 OIL). The laser excitation was set at $405 \mathrm{~nm}$ for Hoechst, and $633 \mathrm{~nm}$ for DiD. The fluorescence emission was set at 410-470 $\mathrm{nm}$ for Hoechst and at $650-750 \mathrm{~nm}$ for DiD.

\section{Statistical Analysis}

All data came from three independent experiments and were presented as mean \pm standard deviation (SD). Student t-test was performed to determine statistical significance between dataset with a level higher than $95 \%$. 


\section{Results and Discussion}

\section{NLC formulation}

The design and formulation of nanostructured lipid carriers (NLC) with three different molar percentages of SA-PEG 100 -GalNAc (SA: stearic acid, for lipid core anchoring; $\mathrm{PEG}_{100}$ : poly(ethylene glycol) spacer with 100 ethylene glycol units; molar percentage: molar $\%$ of SA-PEG ${ }_{100}$-GalNAc compared to total hydrophilic surfactants, Myrj ${ }^{\mathrm{TM}} \mathrm{S} 40$ and SA-PEG $100-$ GalNAc) was already reported [39]. These NLC-GalNAc comprised a lipid core, mix of lecithin, liquid (soybean oil) and solid (Suppocire ${ }^{\mathrm{TM}}$ NB) lipids, and a surfactant shell, composed of Myrj ${ }^{\mathrm{TM}} \mathrm{S} 40$ (mixture of stearate and oleate-poly(ethylene glycol) surfactants with 40 ethylene glycol units) and SA-PEG ${ }_{100}-$ GalNAc ligands. The longer PEG spacer of SA-PEG 100 -GalNAc was expected to present the GalNAc moieties "above" the particle surface, and favor the interactions with ASGPR (Figure 1a). To evaluate the impact of GalNAc grafted at the surface of the NLC on lectin interaction, control NLC were designed. To take into account the potential particle size increase with increasing amount of SA-PEG 100 -GalNAc, NLC with the same molar percentages of Myrjтм S100 ( PEG $_{100}$-based surfactant), (Formulation $\left.A^{\prime}-C^{\prime}\right)$, were formulated as control. SA-PEG $100-$ GalNAc and Myrj ${ }^{\text {TM }}$ S100 both displayed PEG spacers with the same number of ethylene glycol units (100) and were expected to display the same impact on the NLC hydrodynamic diameter.

Interestingly, the accurate quantification of the GalNAc-modified surfactants (resp. Myrj ${ }^{\text {TM }}$ S100) introduced at the NLC surface was achieved by UPLC separation and ELSD detection [39]. This quantification gave access to the effective molar percentages of GalNAc after NLC purification, the mean number of ligands per particle, and the density of ligands that was comprised between 1 GalNAc/32 $\mathrm{nm}^{2}$ (formulation A, $4.6 \%$ 
mol. GalNAc) and 1 GalNAc/17 $\mathrm{nm}^{2}$ (formulation C, 23.3\% mol. GalNAc) (Figure 1a \& Table 2). Detailed procedure for UPLC-ELSD analysis and detailed calculation were reported elsewhere [39].
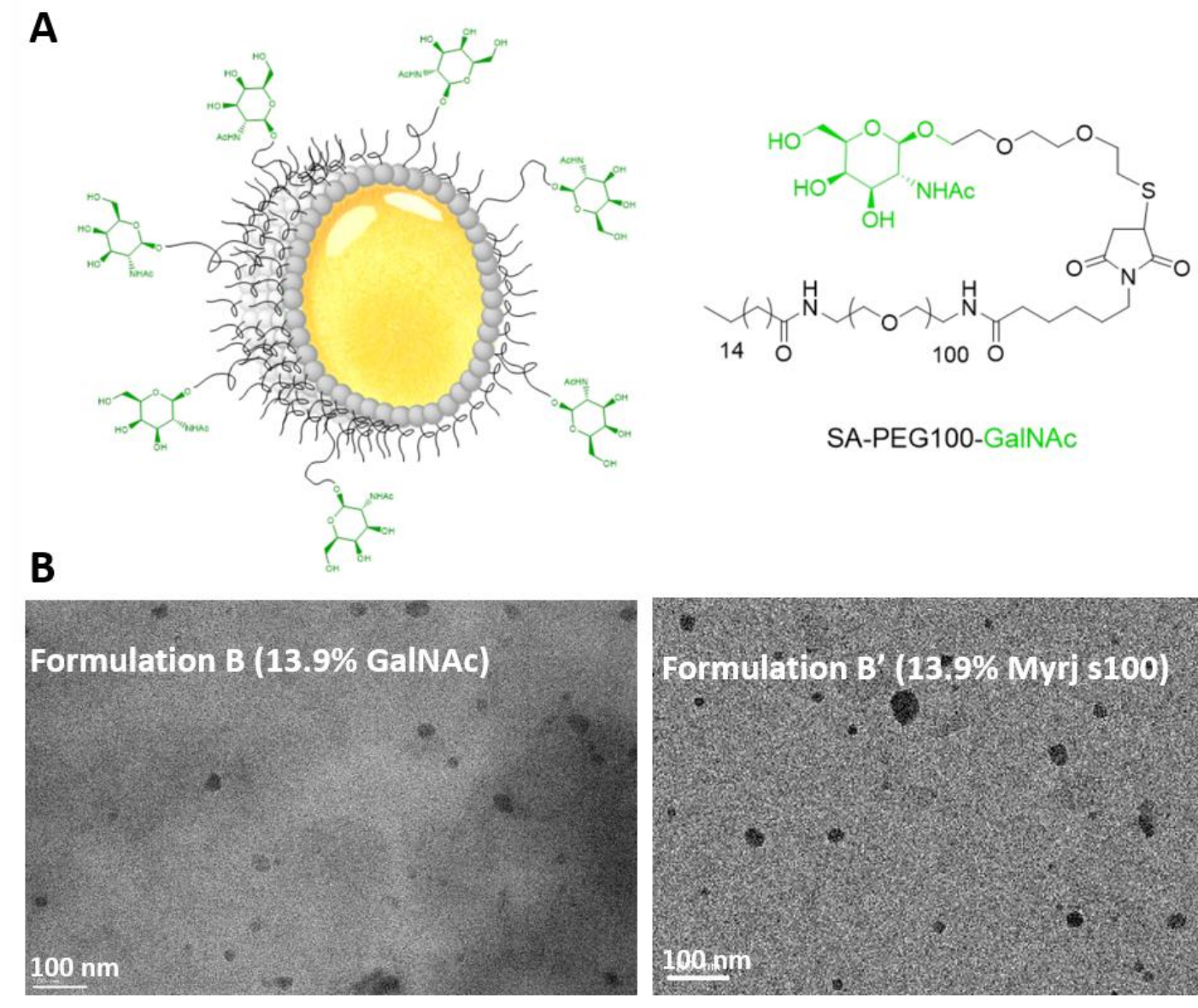

SA-PEG100-GalNAC

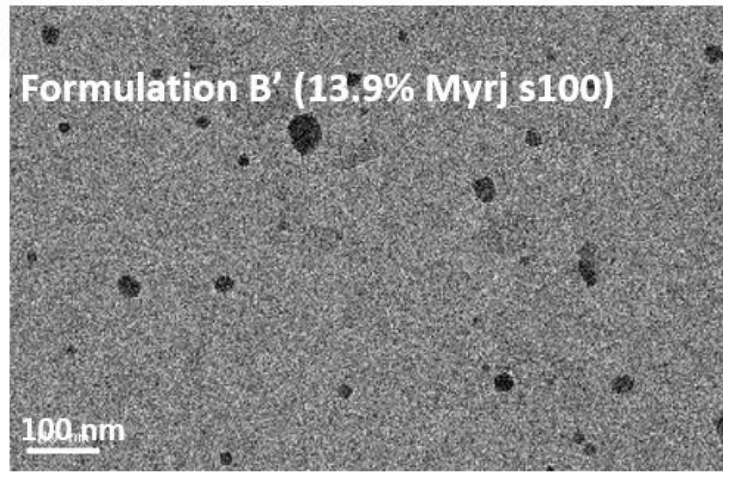

Figure 1. Nanostructured Lipid Carriers (NLC) decorated with GalNAc ligands using SA$\mathrm{PEG}_{100}$-GalNAc as surfactant. a, particle schematic representation, b, TEM images after negative staining of particles from formulations B and B'.

\begin{tabular}{l|r|r|r|}
\hline & \multicolumn{1}{|c|}{ A } & \multicolumn{1}{c}{ B } & \multicolumn{1}{c|}{ C } \\
\hline \% SA-PEG 100 -GalNac & $4.6 \pm 0.05$ & $13.9 \pm 0.04$ & $23.3 \pm 0.01$ \\
\hline SA-PEG $_{100}$-GalNac/Particle & 136 & 333 & 650 \\
\hline Ligand density $\left(/ \mathrm{nm}^{2}\right)$ & $1 / 32$ & $1 / 26$ & $1 / 17$ \\
\hline Average distance between two ligands (nm) & 5.6 & 5.2 & 4.1 \\
\hline
\end{tabular}

Table 2. Quantification of SA-PEG ${ }_{100}-$ GalNAc ligands incorporated at the surface of NLC, density of ligands, and average GalNAc distances for NLC formulated with different molar percentages, based on [39]. 
Formulations A, B, C presenting NLC-GalNAc on their surface indeed displayed the same hydrodynamic diameter and zeta potential than their respectively analogous control formulations A', B', C' incorporating Myrj ${ }^{\mathrm{TM}}$ S100 (Table 3). TEM images confirmed the small nanometric particle size (Figure 1b).

\begin{tabular}{|l|r|r|r|r|r|r|}
\hline & \multicolumn{1}{c|}{$\mathbf{A}$} & \multicolumn{1}{c}{$\boldsymbol{A}^{\prime}$} & \multicolumn{1}{c}{ B } & \multicolumn{1}{c}{$\boldsymbol{B}^{\prime}$} & \multicolumn{1}{c}{ C } & \multicolumn{1}{c}{$\boldsymbol{C}^{\prime}$} \\
\hline Hydrodynamic diameter (nm) & $37 \pm 1$ & $37 \pm 1$ & $52 \pm 1$ & $50 \pm 1$ & $60 \pm 1$ & $64 \pm 1$ \\
\hline PDI & $0.11 \pm 0.03$ & $0.11 \pm 0.03$ & $0.11 \pm 0.02$ & $0.12 \pm 0.01$ & $0.14 \pm 0.02$ & $0.18 \pm 0.02$ \\
\hline Zeta potential (mV) & $-6 \pm 2$ & $-5 \pm 2$ & $-6 \pm 2$ & $-6 \pm 2$ & $-6 \pm 2$ & $-4 \pm 2$ \\
\hline
\end{tabular}

Table 3. Dynamic Light Scattering characterization of NLC formulated with $4.6 \%$ - A, $13.9 \%$ - B and $23.3 \%$ - C of SA-PEG $100-$ GalNAc or $4.6 \%$ - A', 13.9\% - B' and $23.3 \%$ $\mathrm{C}^{\prime}$ of Myrj ${ }^{\mathrm{TM}} \mathrm{S} 100$ in molar percentages. Results (Z-average diameter, polydispersity index, zeta potential) were expressed as mean \pm standard deviation of three independent measurements performed at $25^{\circ} \mathrm{C}$.

Furthermore to follow the putative interaction of NLC with hepatocytes and their subsequent internalization, the DiD fluorophore was encapsulated within the NLC core according to previously published protocols [44]. This lipophilic derivative of cyanine was efficiently encapsulated in the core of the NLC (>95\% for all formulations) without modifying their diameter nor stability. In the NLC, DiD retained its fluorescence properties, limited photobleaching, and did not diffuse through the membrane [44]. All NLC batches were loaded with an equal amount of DiD, and their fluorescence intensity was controlled before each experiment. The different DiD-loaded formulations therefore enabled us to assess the importance of the presence of GalNAc at the surface of the NLC, as well as their density, for the interaction with the ASGPR and the internalization into hepatocytes. 


\section{Hepatocyte uptake of NLC}

Confocal fluorescence microscopy and flow cytometry were used to evaluate the fate of NLC in hepatocytes qualitatively and quantitatively, respectively. For these experiments, the hepatocarcinoma cell line HepG2 and its derivative HepG2/C3a were used. These are standard hepatocyte cellular models that express ASGPR. Confocal fluorescence microscopy was used to analyze the localization of NLC following hepatocyte exposure for 1 hour (Figure 2). GalNAc-NLC and Myrj ${ }^{\mathrm{TM}}$ S100-NLC were observed intracellularly proving that the NLC were rapidly internalized and not bound to plasma membranes. Besides, the staining was punctiform with bunches of assembled nanoparticles, most probably corresponding to endosomal and/or lysosomal localization and confirming that NLC could be endocytosed by hepatocytes.
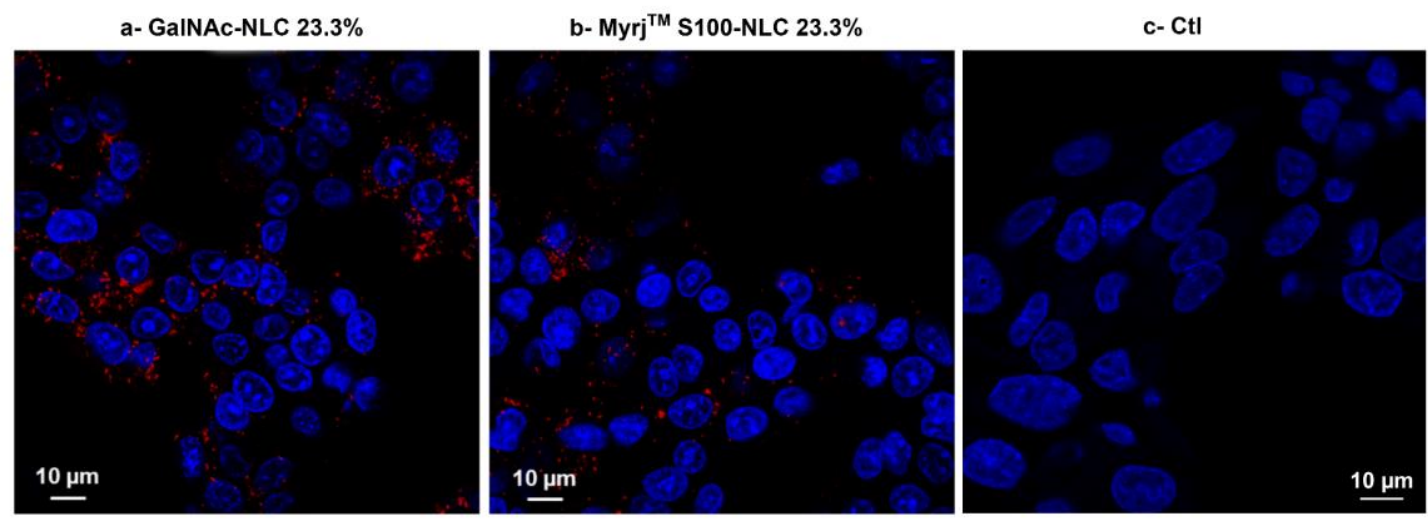

Figure 2. Visualization of NLC cellular uptake in hepatocytes. Confocal fluorescence microscopy of HepG2/C3a cells exposed for 1 hour to DiD-loaded GalNAc-NLC (a) and Myrj ${ }^{\mathrm{TM}} \mathrm{S} 100-\mathrm{NLC}$ (b) in formulation C and C', respectively, and at $500 \mu \mathrm{g} \cdot \mathrm{mL}^{-1}$ in lipids. (c) control cells non exposed to NLC. Nuclei were labeled with Hoechst (blue) and NLC are visualised in red (DiD).

Flow cytometry enables to analyze a whole population cell by cell for the uptake of fluorescent NLC. It was used in our previous studies to compare the internalization of several proprietary glycoconjugates [21-23]. Since an overall endocytosis process is 
performed within minutes, cells were incubated with a series of concentrations of the different NLC formulations for 30 minutes. The tested concentration range was between 0 and $500 \mu \mathrm{g} \cdot \mathrm{mL}^{-1}$ in terms of particle concentration. The fluorescence distribution of cells exposed to a low concentration of GalNAc-NLC (31 $\mu \mathrm{g} \cdot \mathrm{mL}^{-1}$ of lipids) showed a global increase of fluorescence for the whole population of cells compared to cells nonexposed to NLC (Figure S1b vs S1a respectively). Therefore, any cell can endocytose fluorescent NLC, enabling the analysis of the dose-dependent NLC uptake using the MFI of the whole cell population.

The curves representing the MFI as a function of NLC concentration are shown in Figure 3. Each curve displayed a standard hyperbola shape, with a dose dependent increase of the MFI until reaching a plateau. The shape and maximum MFI values were very similar for all curves, except for formulation C, corresponding to higher GalNAc density. Indeed, the MFI was twice those of the other formulations. In particular, the comparison between NLC of formulation C (SA-PEG $100-$ GalNAc) and formulation $\mathrm{C}^{\prime}$ $\left(\mathrm{Myrj}^{\mathrm{TM}} \mathrm{S} 100\right)$, both presenting the same density of $\mathrm{PEG}_{100}$-based surfactants and differing only by the presence of the GalNAc ligand, highlighted this difference in terms of cellular entry (Figure 3). This difference was confirmed by the visual inspection of confocal microscopy images (Figure 2), which showed less NLC spots without GalNAc and no diffuse signal in both cases. This proved that the difference was not due to NLC transformation within hepatocytes, but to a difference in uptake efficiency. Since these two types of NLC presented similar physico-chemical properties (Table 3), it is likely that the high percentage of GalNAc had a significant positive impact on the internalization process of these NLC in hepatocytes. 


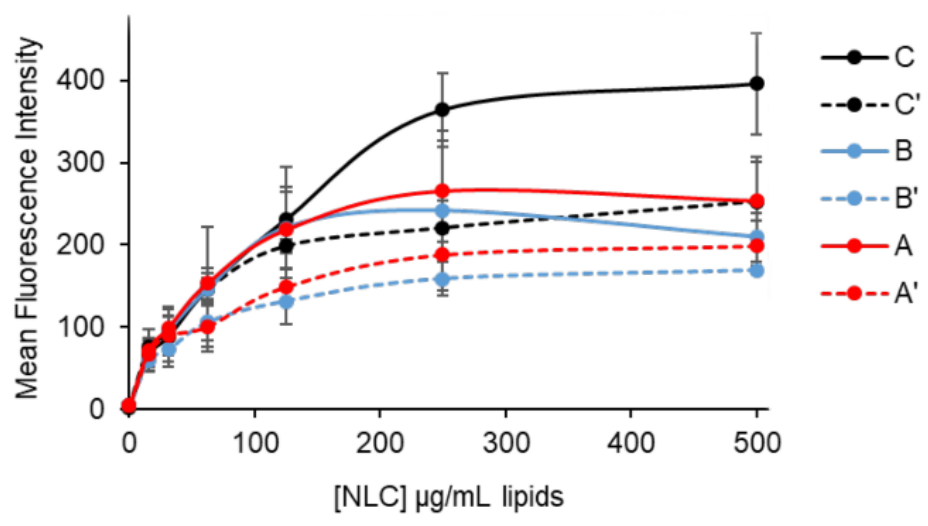

Figure 3. Dose-dependent uptake of NLC in HepG2 cells. Comparison of the entry of DiD-loaded GalNAc-NLC (formulation A, B and C) and Myrj ${ }^{\mathrm{TM}}$ S100-NLC (formulation A', B' and C') at different concentrations after 30 minutes of exposure. The uptake was measured by flow cytometry and the representation corresponded to the MFI of the whole cell population as a function of the NLC concentration for each formulation. Only formulation $\mathrm{C}$ data were significatively different from data of all other formulations with $\mathrm{p}<0.05$.

To confirm this hypothesis and get further information on NLC binding properties, the different curves were fitted using the Hill model (Table 4) in order to determine the half-maximal effective concentration $\left(\mathrm{EC}_{50}\right)$ for hepatocyte NLC uptake in addition to the $\mathrm{MFI}_{\max }$ that we already obtained at $500 \mu \mathrm{g} \cdot \mathrm{mL}^{-1}$ lipids. The $\mathrm{EC}_{50}$ provided an information on the apparent affinity of the NLC for the receptor(s) they used for endocytosis. Besides, the Hill coefficient, that reflects the cooperativity of the interaction, was also optimized in the fit. The $\mathrm{EC}_{50}$ were all comprised between 38 and $47 \mu \mathrm{g} \cdot \mathrm{mL}^{-1}$ at the exception of formulation $\mathrm{C}$ with the highest GalNAc density, for which it was $70 \mu \mathrm{g} \cdot \mathrm{mL}^{-1}$ (Table 4). The difference between formulations $\mathrm{C}$ and C' was significant at more than $95 \%$ confidence and would evidence different sites of interaction at the hepatocyte surface. Altogether, $\mathrm{EC}_{50}$ and MFI analyses showed that the presence of GalNAc units in large amounts favoured a different entry mechanism for the NLC into hepatocytes, most probably via the ASGPR pathway. This result would be consistent with 
the GalNAc average distance between two ligands in formulation C (41 $\mathrm{A})$ approaching the optimal reported value of $20 \AA[16,21-23]$. Unfortunately, it was not possible to decrease the average distance between two GalNAc ligands at the particle surface below $41 \AA$, since it would require important amounts of SA-PEG100-GalNAc and increase the viscosity of the aqueous phase beyond the possible limits of formulation.

\begin{tabular}{l|r|r|r|r|r|r}
\hline & \multicolumn{1}{c|}{ A } & \multicolumn{1}{c|}{$\boldsymbol{A}^{\prime}$} & \multicolumn{1}{c}{ B } & \multicolumn{1}{c|}{$\boldsymbol{B}^{\prime}$} & \multicolumn{1}{c}{ C } & \multicolumn{1}{c}{$\boldsymbol{C}^{\prime}$} \\
\hline Hill Coefficent $n$ & $1.5 \pm 0.6$ & $1.0 \pm 0.2$ & $1.5 \pm 0.5$ & $1.3 \pm 0.6$ & $1.5 \pm 0.6$ & $1.7 \pm 0.7$ \\
\hline EC50 $(\mu \mathrm{g} / \mathrm{mL})$ & $43 \pm 7$ & $47 \pm 6$ & $41 \pm 4$ & $44 \pm 10$ & $70 \pm 15$ & $45 \pm 20$ \\
\hline
\end{tabular}

Table 4: Analysis of dose-dependent NLC hepatocyte uptake curves (Figure 3) using the Hill equation: $y=\frac{y_{\max } * x^{n}}{\left(E C_{50}^{n}+x^{n}\right)}$ where $\mathrm{y}$ is the fluorescence intensity, $\mathrm{x}$ is the NLC concentration in $\mu \mathrm{g} \cdot \mathrm{mL}^{-1}$ and $\mathrm{n}$ is the Hill coefficient. The data points used to draw the curves correspond to the average from three independent experiments. Values and standard deviations obtained for Hill coefficients and $\mathrm{EC}_{50}$ were obtained by fitting using the Hill equation.

\section{Molecular interaction between GalNAc-NLC and the macrophage galactose-lectin}

In order to confirm that NLC uptake by hepatocytes was dependent on ASGPR, the molecular interaction of GalNAc-NLC with such receptors was an important control. Recombinant ASGPR production is known as being a difficult task, notably due to the need to reconstitute it from different chains with specific stoichiometry. Thus, here we used another lectin that can be considered as a good model of ASGPR for this kind of characterization, the MGL. As ASGPR, MGL is a C-Type lectin with a CRD specific of Gal and GalNAc recognition in a $\mathrm{Ca}^{2+}$-dependent manner. MGL recognizes more particularly the tumor associated Tn antigen (Thomsen-nouveau), a truncated O-glycan with surface-exposed GalNAc units [48]. Moreover, it also forms oligomers but MGL are strictly homotrimers, formed from a unique polypeptide chain $[49,50]$. Thus, MGL is 
more accessible to recombinant production and is routinely produced in our laboratory [46]. It has been used here for molecular interaction experiments with GalNAc-NLC. SPR experiments were performed to investigate the ability of GalNAc decorated NLC to interact at the molecular level with MGL, used as a model of GalNAc-recognizing lectins. The refolded trimeric extracellular domain of the MGL was used to prepare SPR CM3 sensor chips [46]. The functionality and the specificity of the surface were assessed with BSA-GalNAc and BSA-Mannose (as a negative control) that are commonly used models for multivalent binding. While monovalent GalNAc have a reported affinity in the micromolar range $(12 \mu \mathrm{M})$ for MGL [51], the apparent affinity of BSA-GalNAc for the MGL surface was in the high nanomolar range $(8 \mathrm{nM})$ (Figure S2a-b), while BSAmannose did not bind to the MGL surface (Figure S2c). This validated the quality and the specificity of the surface, and in particular confirmed the possibility to detect multiple binding (avidity).

Negative signals of the reference surface corrected sensorgrams were observed during the association phase of NLC with these sensor chips (CM3). This was probably due to non-specific interactions between dextran that covers those sensor chips and the PEG of the NLC. Despite this effect, it was possible to observe a binding tendency to the MGL surface when GalNAc was present at the NLC surface (Figure S3a versus S3b). Besides, none of the NLC were binding to the DC-SIGN surface, a lectin that recognizes mannose (Figure S3c-d). Therefore, the decoration of NLC with GalNAc enabled the specific recruitment by GalNAc-binding lectins.

To solve the issue observed with CM3 sensor chips, experiments were performed with $\mathrm{C} 1$ sensor chips that are not covered by dextran. The positive control with BSAGalNAc was reconducted on these sensor chips and confirmed their functionality (Figure $\mathbf{S 4}, \mathrm{K}_{\mathrm{d} \text {-app }}=38 \mathrm{nM}$ ). Besides, the non-specific binding of NLC was thus highly reduced 
on these $\mathrm{C} 1$ sensor chips and the double subtracted data showed very nice binding association and dissociation curves for GalNAc-NLC formulation C (23.3\% mol., Figure 4a), while the corresponding control NLC (formulation C') did not show any binding for the same concentration of lipids (Figure 4b). Interestingly, the decrease of GalNAc density led to a decrease of interaction with the MGL surface for formulation B (13.9\% mol., Figure 4c) and no specific interaction for formulation A (4.6\% mol., Figure 4e). The corresponding NLC controls, formulation A' and B' did not show any binding (Figure 4d and 4f) and A and A' presented very similar double subtracted sensorgrams (Figure 4e and $\mathbf{4 f}$ ). These results were consistent with the cellular data that showed the requirement of a high amount of GalNAc per NLC for recruitment by lectins and thereof to induce hepatocyte endocytosis via the ASGPR pathway. This experiment also leads to questions concerning GalNAc location and orientation within NLC formulations.

Since the data did not follow a simple one-to-one association model it was not possible to accurately determine an apparent affinity but it was in the order of several tens of micromolar. Therefore, there seemed to be no or low avidity involved in the interaction between the GalNAc-NLC and MGL, while it was the case with BSA-GalNAc. It suggests that GalNAc moieties, despite being present in large amount within the NLC, are not sufficiently accessible to allow avidity phenomenon. Further developments, addressing this concern, will undoubtedly allow to largely improve NLC targeting thanks to avidity potentiation. It is interesting to notice that the SPR strategy was efficient to assess the potential binding to lectins, and to confirm the mechanism of cellular endocytosis of glycoconjugated-NLC. 

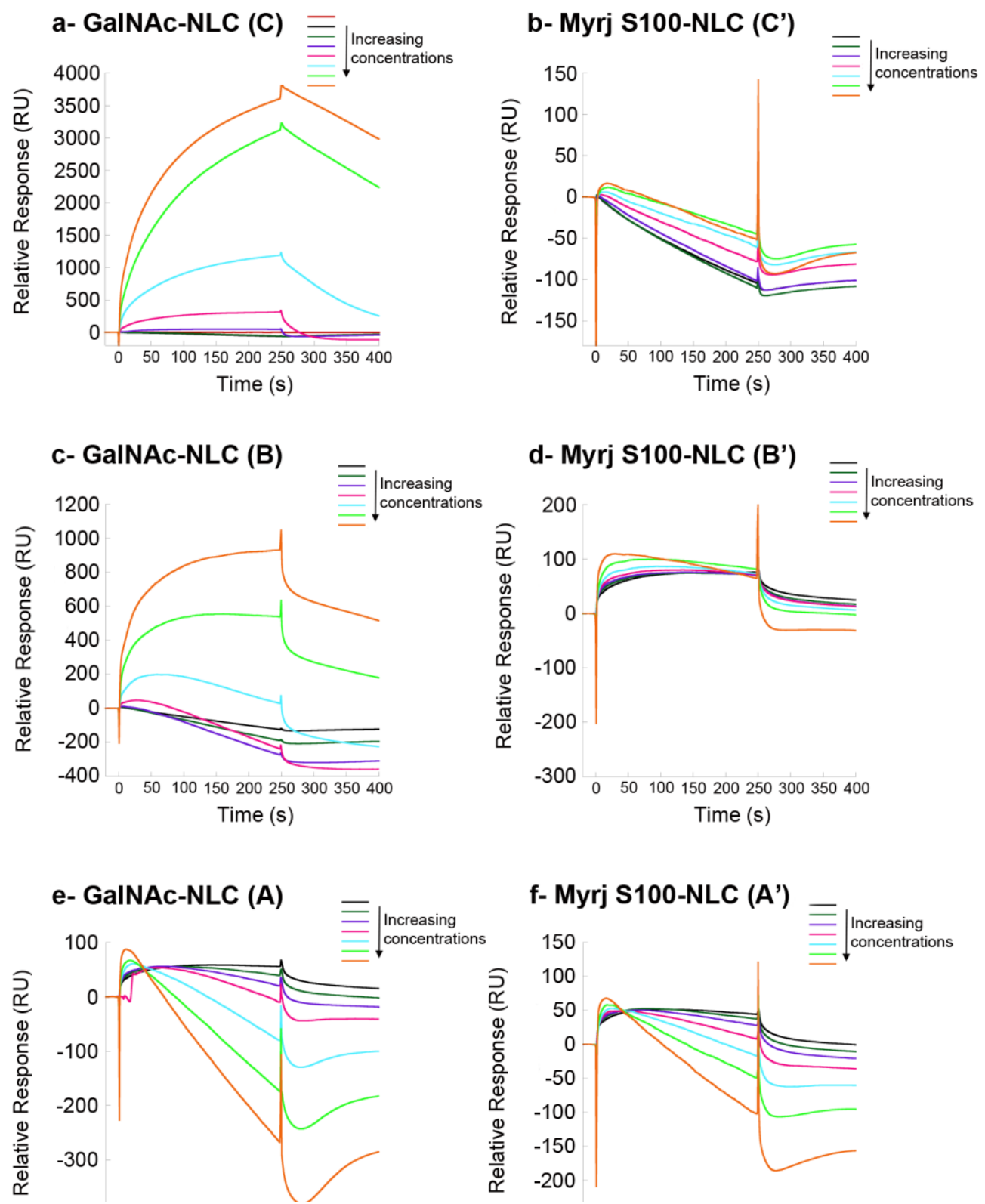

Figure 4. NLC binding to MGL C1 surfaces. Reference surface corrected sensorgrams upon injection of increasing concentrations of GalNAc-NLC, a, formulation C, $23.3 \%$, c formulation B, $13.9 \%$ and e, formulation A, 4.6\%, over MGL-functionalized surface. Reference surface corrected sensorgrams upon injection of increasing concentrations of $\mathrm{Myrj}^{\mathrm{TM}} \mathrm{S} 100-\mathrm{NLC}$ b, formulation C', $23.3 \%$, d, formulation B', $13.9 \%$ and $\mathrm{f}$, formulation A', 4.6\%, over MGL-functionalized surface. For all experiments, 2-fold dilution series were used with a starting concentration of $250 \mu \mathrm{M}$ in GalNAc for GalNAc-NLC and the equivalent lipid concentration for Myrj ${ }^{\mathrm{TM}}$ S100-NLC. Each value corresponds to the average and standard deviation of three independent experiments. 


\section{Conclusion}

In this study, we assessed the influence of the molar percentage of GalNAc in NLC formulation to target the ASGPR pathway for hepatocyte endocytosis. Indeed, this receptor is abundant at the surface of this cell type and is therefore an interesting option to specifically deliver a drug to the liver. As for other types of nanoparticles, NLC were internalized by hepatocytes but high density of GalNAc was required to favor the endocytosis process via the ASGPR pathway. Indeed, SPR experiments using the MGL, another GalNAc-binding lectin, proved at the molecular level that a high density of GalNAc was a prerequisite for efficient NLC binding to specific lectins. Molecular and cellular experiments were thus consistent and showed the efficiency of NLC with high density of GalNAc for specific liver targeting, most probably mediated by ASGPR. The low affinity between a single GalNAc and a lectin binding site explains the requirement for high GalNAc density. Based on our previous developments, it could be interesting to graft multi-GalNAc moieties, such as tri-antenna, at the extremity of the PEG chains. This strategy would help to increase avidity and thus the efficiency of the endocytic process with respect to the amount of GalNAc units per NLC. Ultimately, it could improve the delivery to the liver of active substances encapsulated in the core of the lipid nanoparticles.

\section{Acknowledgements}

We thank Veronique Collin-Faure for her support for flow cytometry experiments and Daphna Fenel (ISBG, Univ. Grenoble Alpes, France) for the acquisition of TEM images. This work used the EM facilities at the Grenoble Instruct-ERIC Center (ISBG; UMS 3518 CNRS CEA-UGA-EMBL) with support from the French Infrastructure for Integrated

Structural Biology (FRISBI; ANR-10-INSB-05-02) and GRAL, a project of the 
University Grenoble Alpes graduate school (Ecoles Universitaires de Recherche) CBHEUR-GS (ANR-17-EURE-0003) within the Grenoble Partnership for Structural Biology. The IBS Electron Microscope facility is supported by the Auvergne Rhône-Alpes Region, the Fonds Feder, the Fondation pour la Recherche Médicale and GIS-IBiSA.

\section{Disclosure statement}

No potential conflict of interest was reported by the authors.

\section{Funding}

This work was supported by the Région Auvergne Rhône-Alpes (Ph D grant for L.G.) and the French National Research Agency in the framework of LabEx Arcane and CBH-EUR-GS (grant ANR-17-EURE-0003), and Glyco@Alps “Investissement d'avenir” program (ANR-15-IDEX$02)$.

\section{References}

[1] Ashwell G, Harford J. Carbohydrate-Specific Receptors of the Liver. Annu Rev Biochem. 1982;51:531-554.

[2] Spiess M. The asialoglycoprotein receptor: a model for endocytic transport receptors. Biochemistry (Mosc). 1990;29:10009-10018.

[3] Das S, Kudale P, Dandekar P, et al. Asialoglycoprotein Receptor and Targeting Strategies. In: Devarajan PV, Dandekar P, D'Souza AA, editors. Target Intracell Drug Deliv Recept Mediat Endocytosis [Internet]. Cham: Springer International Publishing; 2019 [cited 2020 Apr 8]. p. 353-381. Available from: http://link.springer.com/10.1007/978-3-030-29168-6_12.

[4] D'Souza AA, Devarajan PV. Asialoglycoprotein receptor mediated hepatocyte targeting - Strategies and applications. J Controlled Release. 2015;203:126-139.

[5] Cummings RD, McEver RP. C-type Lectins. In: Varki A, Cummings RD, Esko JD, et al., editors. Essent Glycobiol [Internet]. 2nd ed. Cold Spring Harbor (NY): Cold Spring Harbor Laboratory Press; 2009 [cited 2020 Apr 3]. Available from: http://www.ncbi.nlm.nih.gov/books/NBK1943/.

[6] Meier M, Bider MD, Malashkevich VN, et al. Crystal Structure of the Carbohydrate Recognition Domain of the H1 Subunit of the Asialoglycoprotein Receptor. J Mol Biol. 2000;300:857-865.

[7] Connolly DT, Townsend RR, Kawaguchi K, et al. Binding and endocytosis of cluster glycosides by rabbit hepatocytes. Evidence for a short-circuit pathway that does not lead to degradation. J Biol Chem. 1982;257:939-945. 
[8] Baenziger JU, Maynard Y. Human hepatic lectin. Physiochemical properties and specificity. J Biol Chem. 1980;255:4607-4613.

[9] Hardy MR, Townsend RR, Parkhurst SM, et al. Different modes of ligand binding to the hepatic galactose/ $\mathrm{N}$-acetylgalactosamine lectin on the surface of rabbit hepatocytes. Biochemistry (Mosc). 1985;24:22-28.

[10] Huang X, Leroux J-C, Castagner B. Well-Defined Multivalent Ligands for Hepatocytes Targeting via Asialoglycoprotein Receptor. Bioconjug Chem. 2017;28:283-295.

[11] Sanhueza CA, Baksh MM, Thuma B, et al. Efficient Liver Targeting by Polyvalent Display of a Compact Ligand for the Asialoglycoprotein Receptor. J Am Chem Soc. 2017;139:3528-3536.

[12] Nair JK, Willoughby JLS, Chan A, et al. Multivalent $N$-AcetylgalactosamineConjugated siRNA Localizes in Hepatocytes and Elicits Robust RNAi-Mediated Gene Silencing. J Am Chem Soc. 2014;136:16958-16961.

[13] Majouga A, Ivanenkov $\mathrm{Y}$, Veselov $\mathrm{M}$, et al. Identification of Novel SmallMolecule ASGP-R Ligands. Curr Drug Deliv. 2016;13:1303-1312.

[14] Prakash TP, Yu J, Migawa MT, et al. Comprehensive Structure-Activity Relationship of Triantennary $N$-Acetylgalactosamine Conjugated Antisense Oligonucleotides for Targeted Delivery to Hepatocytes. J Med Chem. 2016;59:2718-2733.

[15] Prakash TP, Graham MJ, Yu J, et al. Targeted delivery of antisense oligonucleotides to hepatocytes using triantennary $N$-acetyl galactosamine improves potency 10-fold in mice. Nucleic Acids Res. 2014;42:8796-8807.

[16] Khorev O, Stokmaier D, Schwardt O, et al. Trivalent, Gal/GalNAc-containing ligands designed for the asialoglycoprotein receptor. Bioorg Med Chem. 2008;16:5216-5231.

[17] Westerlind U, Westman J, Törnquist E, et al. Ligands of the asialoglycoprotein receptor for targeted gene delivery, part 1: Synthesis of and binding studies with biotinylated cluster glycosides containing N-acetylgalactosamine. Glycoconj J. 2004;21:227-241.

[18] Rensen PCN, Sliedregt LAJM, Ferns M, et al. Determination of the Upper Size Limit for Uptake and Processing of Ligands by the Asialoglycoprotein Receptor on Hepatocytes in Vitro and in Vivo. J Biol Chem. 2001;276:37577-37584.

[19] Lee YC, Townsend RR, Hardy MR, et al. Binding of synthetic oligosaccharides to the hepatic Gal/GalNAc lectin. Dependence on fine structural features. J Biol Chem. 1983;258:199-202.

[20] Gateau C, Delangle P. Design of intrahepatocyte copper(I) chelators as drug candidates for Wilson's disease: Intracellular copper chelation. Ann N Y Acad Sci. 2014;1315:30-36. 
[21] Monestier M, Charbonnier P, Gateau C, et al. ASGPR-Mediated Uptake of Multivalent Glycoconjugates for Drug Delivery in Hepatocytes. Chembiochem Eur J Chem Biol. 2016;17:590-594.

[22] Pujol AM, Cuillel M, Jullien A-S, et al. A sulfur tripod glycoconjugate that releases a high-affinity copper chelator in hepatocytes. Angew Chem Int Ed Engl. 2012;51:7445-7448.

[23] Pujol AM, Cuillel M, Renaudet O, et al. Hepatocyte targeting and intracellular copper chelation by a thiol-containing glycocyclopeptide. J Am Chem Soc. 2011;133:286-296.

[24] Zhu C, Zheng Q, Wang L, et al. Synthesis of novel galactose functionalized gold nanoparticles and its radiosensitizing mechanism. J Nanobiotechnology [Internet]. 2015 [cited $2020 \quad$ Apr 1];13. Available from: http://www.jnanobiotechnology.com/content/13/1/67.

[25] Morille M, Passirani C, Letrou-Bonneval E, et al. Galactosylated DNA lipid nanocapsules for efficient hepatocyte targeting. Int J Pharm. 2009;379:293-300.

[26] Wang T, Tang X, Han J, et al. Biodegradable Self-Assembled Nanoparticles of Galactose-Containing Amphiphilic Triblock Copolymers for Targeted Delivery of Paclitaxel to HepG2 Cells. Macromol Biosci. 2016;16:774-783.

[27] Managit C, Kawakami S, Yamashita F, et al. Effect of Galactose Density on Asialoglycoprotein Receptor-Mediated Uptake of Galactosylated Liposomes. J Pharm Sci. 2005;94:2266-2275.

[28] Kawakami S, Munakata C, Fumoto S, et al. Novel Galactosylated Liposomes for Hepatocyte-Selective Targeting of Lipophilic Drugs. J Pharm Sci. 2001;90:105113.

[29] Oh H, Jo H-Y, Park J, et al. Galactosylated Liposomes for Targeted Co-Delivery of Doxorubicin/Vimentin siRNA to Hepatocellular Carcinoma. Nanomaterials. 2016;6:141.

[30] Sato Y, Kinami Y, Hashiba K, et al. Different kinetics for the hepatic uptake of lipid nanoparticles between the apolipoprotein E/low density lipoprotein receptor and the N-acetyl-d-galactosamine/asialoglycoprotein receptor pathway. J Controlled Release. 2020;322:217-226.

[31] Akinc A, Querbes W, De S, et al. Targeted Delivery of RNAi Therapeutics With Endogenous and Exogenous Ligand-Based Mechanisms. Mol Ther. 2010;18:1357-1364.

[32] Huang Y. Preclinical and Clinical Advances of GalNAc-Decorated Nucleic Acid Therapeutics. Mol Ther - Nucleic Acids. 2017;6:116-132.

[33] Carbone C, Leonardi A, Cupri S, et al. Pharmaceutical and biomedical applications of lipid-based nanocarriers. Pharm Pat Anal. 2014;3:199-215. 
[34] Teixeira MC, Carbone C, Souto EB. Beyond liposomes: Recent advances on lipid based nanostructures for poorly soluble/poorly permeable drug delivery. Prog Lipid Res. 2017;68:1-11.

[35] Doktorovová S, Kovačević AB, Garcia ML, et al. Preclinical safety of solid lipid nanoparticles and nanostructured lipid carriers: Current evidence from in vitro and in vivo evaluation. Eur J Pharm Biopharm. 2016;108:235-252.

[36] H. Muller R, Shegokar R, M. Keck C. 20 Years of Lipid Nanoparticles (SLN \& NLC): Present State of Development \& Industrial Applications. Curr Drug Discov Technol. 2011;8:207-227.

[37] Sawant K, Dodiya S. Recent Advances and Patents on Solid Lipid Nanoparticles. Recent Pat Drug Deliv Formul. 2008;2:120-135.

[38] Hattori Y, Kawakami S, Yamashita F, et al. Controlled biodistribution of galactosylated liposomes and incorporated probucol in hepatocyte-selective drug targeting. J Controlled Release. 2000;69:369-377.

[39] Gauthier, Varache, Couffin, et al. Quantification of Surface GalNAc Ligands Decorating Nanostructured Lipid Carriers by UPLC-ELSD. Int J Mol Sci. 2019;20:5669.

[40] Beloqui A, Solinís MÁ, Rodríguez-Gascón A, et al. Nanostructured lipid carriers: Promising drug delivery systems for future clinics. Nanomedicine Nanotechnol Biol Med. 2016;12:143-161.

[41] Müller R., Radtke M, Wissing S. Nanostructured lipid matrices for improved microencapsulation of drugs. Int J Pharm. 2002;242:121-128.

[42] Vigne J, Cabella C, Dézsi L, et al. Nanostructured lipid carriers accumulate in atherosclerotic plaques of ApoE-/- mice. Nanomedicine Nanotechnol Biol Med. 2020;25:102157.

[43] Delmas T, Couffin A-C, Bayle PA, et al. Preparation and characterization of highly stable lipid nanoparticles with amorphous core of tuneable viscosity. J Colloid Interface Sci. 2011;360:471-481.

[44] Gravier J, Navarro FP, Delmas T, et al. Lipidots: competitive organic alternative to quantum dots for in vivo fluorescence imaging. J Biomed Opt. 2011;16:096013.

[45] Caputo F, Arnould A, Bacia M, et al. Measuring Particle Size Distribution by Asymmetric Flow Field Flow Fractionation: A Powerful Method for the Preclinical Characterization of Lipid-Based Nanoparticles. Mol Pharm. 2019;16:756-767.

[46] Maalej MM, Forgione RE, Marchetti R, et al. The human macrophage galactosetype lectin, MGL, recognizes the outer core of E. coli lipooligosaccharide. ChemBioChem [Internet]. 2019 [cited 2020 Apr 4]; Available from: https://onlinelibrary.wiley.com/doi/abs/10.1002/cbic.201900087. 
[47] Porkolab V, Pifferi C, Sutkeviciute I, et al. Development of C-type lectin-oriented surfaces for high avidity glycoconjugates: towards mimicking multivalent interactions on the cell surface. Org Biomol Chem. 2020;18:4763-4772.

[48] Fu C, Zhao H, Wang Y, et al. Tumor-associated antigens: Tn antigen, sTn antigen, and $\mathrm{T}$ antigen: Tumor markers related to treatment. HLA. 2016;88:275-286.

[49] Jégouzo SA, Quintero-Martínez A, Ouyang X, et al. Organization of the extracellular portion of the macrophage galactose receptor: A trimeric cluster of simple binding sites for N-acetylgalactosamine. Glycobiology. 2013;23:853-864.

[50] Diniz A, Coelho H, Dias JS, et al. The Plasticity of the Carbohydrate Recognition Domain Dictates the Exquisite Mechanism of Binding of Human Macrophage Galactose-Type Lectin. Chem - Eur J. 2019;25:13945-13955.

[51] Marcelo F, Garcia-Martin F, Matsushita T, et al. Delineating Binding Modes of Gal/GalNAc and Structural Elements of the Molecular Recognition of TumorAssociated Mucin Glycopeptides by the Human Macrophage Galactose-Type Lectin. Chem - Eur J. 2014;20:16147-16155. 